\title{
EFECTO DE LA SUPLEMENTACIÓN CON BLOQUES MINERALES SOBRE LA PRODUCTIVIDAD DE CUYES ALIMENTADOS CON FORRAJE
}

\author{
Effect of Mineral Block Supplementation on the Productive Performance \\ of Forage-Fed Guinea Pigs
}

\section{Carlos Castillo G. ${ }^{1}$, Fernando Carcelén C. ${ }^{1,3}$, William Quevedo G. ${ }^{2}$, Miguel Ara G. ${ }^{1}$}

\section{RESUMEN}

El objetivo del presente estudio fue evaluar el efecto de la suplementación con bloques minerales sobre los parámetros productivos en cuyes de engorde alimentados con maíz chala en condiciones de la costa central de Lima, Perú. Se emplearon 32 cuyes machos, recién destetados, de la raza Perú, distribuidos en ocho pozas de crianza. Se empleó un diseño completamente al azar con dos tratamientos con cuatro repeticiones (las pozas). Los tratamientos fueron T0 [alimentación con forraje (maíz chala)] y T1 (alimentación con forraje y suplementación con bloques conteniendo macro y microminerales). Se evaluó ganancia de peso, consumo de alimento, conversión alimenticia y mérito económico a las 12 semanas del estudio. Se encontró diferencia significativa en ganancia de peso (T0: 358.8 y T1: 476.7 g) y en conversión alimenticia (T0: 6.9 y T1: 5.5) $(\mathrm{p}<0.05)$, pero no hubo diferencia estadística en el consumo de materia seca. La producción de 100 g de peso vivo de cuy fue $9 \%$ más económica con el tratamiento T1. Se concluye que la suplementación con bloques minerales tiene potencial para incrementar la productividad del cuy en crianzas en condiciones de la costa central peruana.

Palabras clave: cuy, suplemento mineral, bloques, alimentación con forraje

\section{Abstract}

The purpose of this study was to evaluate the effect of mineral block supplementation on the productive performance of guinea pigs in a forage-based rearing system under the Peruvian Central Coastal conditions. Thirty two 15-day weaned, male, Peru breed guinea pigs were randomly assigned to eight rearing boxes. A completely random design with two treatments and four replications was used in the study. Treatments were T0 (feeding with maize forage) and T1 (as T0 plus free access to blocks containing macro and

\footnotetext{
${ }^{1}$ Laboratorio de Bioquímica, Nutrición y Alimentación Animal, ${ }^{2}$ Laboratorio de Zootecnia y Producción Agropecuaria, Facultad de Medicina Veterinaria, Universidad Nacional Mayor de San Marcos, Lima

${ }^{3}$ E-mail: nando2910@hotmail.com
} 
microminerals). Body weight gain, feed intake, feed conversion and the economic merit were evaluated. There was statistical difference between treatments on body weight gain (T0: 358.8 and T1: $476.7 \mathrm{~g}$ ) and feed conversion index (T0: 6.9 and T1: 5.5) ( $\mathrm{p}<0.05)$, but without statistical difference on feed intake. It was $9 \%$ cheaper to produce $100 \mathrm{~g}$ body weight of guinea pig using mineral blocks. It is concluded that the use of mineral blocks has a potential to increase guinea pig productivity in the forage-based rearing system of the Peruvian Central Coast.

Key words: guinea pig, mineral supplementation, mineral blocks, forage feeding

\section{INTRODUCCIÓN}

El cuy es una especie herbívora monogástrica, con un estómago que inicia la digestión enzimática y un ciego que realiza la fermentación bacteriana. Junto al conejo, rata y capibara, el cuy está clasificado por su anatomía gastrointestinal como fermentador postgástrico cecal, dada la importancia de la actividad microbiana en el ciego para los procesos de digestión y utilización de nutrientes (Chauca, 1994). Esta característica le confiere una gran eficacia en la digestión de la fibra y sus componentes, eficacia comparable a los caballos (Hirikawa, 2001).

El aprovechamiento del material fibroso permite la utilización del cuy en sistemas de producción pecuaria en base al suministro casi exclusivo de forrajes (Chauca, 1997). Sin embargo, la respuesta productiva depende, en parte, del contenido de minerales y vitaminas en el forraje, el cual es altamente variable, dependiendo de la especie forrajera, edad de corte, estado fenológico, disponibilidad de humedad y calidad del suelo.

Una posibilidad de mejorar la calidad de la dieta es el uso de bloques nutricionales, los cuales son usualmente elaborados con subproductos de cosecha y cereales, derivados de la agroindustria, fuentes sintéticas de $\mathrm{N}$ como urea, minerales, y aglomerantes compactados a presión (Becerra y David,
1990; Rivadeneyra, 1999). Aunque esta metodología se encuentra bastante difundida, especialmente en rumiantes al pastoreo (Vargas y Rivera, 1994; Rueda y de Combellas, 1999), la experiencia en cuyes es aún incipiente. Se dispone de la experiencia del uso de bloques multinutricionales frente al uso de concentrados comerciales con dietas forrajeras a base de kikuyo (Pennisetum clandestinum) o Lolium multiflorum en Colombia (Díaz, 1990), y con afrechillo o concentrado comercial bajo una dieta forrajera de alfalfa (Medicago sativa) en el Perú (Rivadeneyra, 1999), donde la respuesta del bloque fue similar a los concentrados comerciales.

En la costa central de Perú, los sistemas de crianza de cuyes usan mayormente el maíz chala (Zea mays) como forraje debido a su bajo costo y mayor disponibilidad. Esta especie, sin embargo, tiene un limitado aporte de nutrientes comparada con otras como la alfalfa (Saravia, 1994) y, bajo un sistema de crianza basado casi exclusivamente en forraje, es probable que se generen deficiencias minerales, tanto por el bajo valor nutritivo del maíz chala como por la reducida ingestión de materia seca. Considerando que los bloques nutricionales podrían ser utilizados como vehículos para el aporte mineral, el objetivo del presente estudio fue determinar la efectividad de bloques minerales sobre el comportamiento productivo y mérito económico de cuyes alimentados con forraje en condiciones de la costa central peruana. 
Cuadro 1. Composición de los bloques minerales empleados en el ensayo

\begin{tabular}{lc}
\hline Ingredientes & $\%$ \\
\hline Ingredientes activos & \\
Fosfato dicálcico & 50.0 \\
Sulfato cúprico & 0.48 \\
Óxido de manganeso & 0.0968 \\
Óxido de zinc & 0.92 \\
Ioduro de potasio & 0.0116 \\
Sulfato de cobalto & 0.0238 \\
Selenito de sodio & 0.0055 \\
Óxido de magnesio & 1.667 \\
Transportadores & \\
Sal (cloruro de sodio) & 14.8 \\
Linaza & 5.0 \\
Aglomerantes & \\
Cemento & 20.0 \\
Cal viva & 7.0 \\
Total: & 100.0 \\
\hline
\end{tabular}

Cuadro 2. Aporte de nutrientes minerales de los bloques usados en el ensayo

\begin{tabular}{lr}
\hline Aporte de minerales & \\
\hline Macrominerales $(\%)$ & \\
Cal cio & 20.4 \\
Fósforo & 9.0 \\
Sodio & 5.8 \\
Microminerales (mg/1000 g) & \\
Magnesio & 1200.0 \\
Cobalto & 50.0 \\
Zinc & 6.7 \\
Manganeso & 600.0 \\
Yodo & 80.0 \\
Selenio & 25.0 \\
\hline
\end{tabular}

\section{MATERIALES y Métodos}

Se utilizaron 32 cuyes machos recién destetados de la raza Perú, con identificación individual y con un peso promedio de
206 g, pertenecientes a la granja comercial "Centinela" (km 60 de la Panamericana Sur, distrito de Pucusana, Lima). El trabajo se realizó entre marzo y julio de 2007 y el destete se realizó a los 15 días de edad.

Los animales fueron aleatoriamente distribuidos en ocho pozas de cemento de $0.9 \mathrm{x}$ $0.75 \times 0.45 \mathrm{~m}$, dentro de un galpón de ladrillo y cemento de $400 \mathrm{~m}^{2}$. Las pozas fueron asignadas al azar en dos tratamientos: T0, alimentación con forraje (maíz chala), y T1, como en T0 pero suplementados con bloques minerales.

Se emplearon bloques comerciales (Corporación Aygut SA), cilíndricos, de $11 \mathrm{~cm}$ de diámetro y $2.5 \mathrm{~cm}$ de alto, compuestos de ingredientes activos, transportadores y aglomerantes (Cuadro 1). El aporte mineral del bloque se muestra en el Cuadro 2.

Previo al experimento, las pozas fueron limpiadas, desinfectadas, provistas con una cama de paja de arroz de $10 \mathrm{~cm}$ de altura y equipadas con bebederos rectangulares con agua enriquecida con vitamina C. Durante el

Cuadro 3. Valores promedio del análisis proximal $^{1} \mathrm{y}$ del conteni do de minerales del maíz chala (Zea mays) usado en el experimento (en base al $100 \%$ de la materia seca)

\begin{tabular}{lr}
\hline Parámetros & $\%$ \\
\hline Proximal & \\
Proteina & 14.6 \\
Extracto etéreo & 2.2 \\
Fibra cruda & 30.1 \\
Extracto no nitrogenado & 45.0 \\
Ceniza & 8.1 \\
Minerales & \\
Calcio & 1.3 \\
Fósforo & 0.5 \\
\hline
\end{tabular}

${ }^{1}$ El contenid o de materia seca fue de $16.6 \%$ 
Cuadro 4. Ganancia de peso, consumo e indice de conversión alimenticia promedio en cuyes de la raza Perú, alimentados con forraje maíz chala (Zea mays) y supl ementados con bloques minerales ( 16 cuyes por tratami ento)

\begin{tabular}{lcc}
\hline \multirow{2}{*}{ Parámetros produ divos } & \multicolumn{2}{c}{ Dieta básica de maíz chala adlibitum } \\
\cline { 2 - 3 } & Sin suplemento & Con bloque mineral \\
\hline Peso inicial $(\mathrm{g})$ & $209.4^{\mathrm{a}}$ & $203.7^{\mathrm{a}}$ \\
Ganancia total de peso $(\mathrm{g})$ & $358.8^{\mathrm{a}}$ & $476.7^{\mathrm{b}}$ \\
Con sumo total de materia seca $(\mathrm{g})$ & $2492.0^{\mathrm{a}}$ & $2633.9^{\mathrm{a}}$ \\
Índice de oo nver són alimenticia & $6.9^{\mathrm{a}}$ & $5.5^{\mathrm{b}}$ \\
\hline
\end{tabular}

${ }^{\text {ab }}$ Superíndices diferentes dentro de filas indican dferencias significativas $(\mathrm{p}<0.05)$ entre tratamientos

experimento, el forraje fue adquirido dos veces por semana de un proveedor local, y fue suministrado ad libitum a las 09:00 y a las 16:00. El alimento fue colocado en el piso de las pozas. El consumo del forraje fue calculado por diferencia entre lo ofrecido y lo rechazado. La composición del maíz chala utilizado se presenta en el Cuadro 3.

La respuesta a la suplementación mineral se evaluó en términos de ganancia de peso, índice de conversión alimenticia y mérito económico. Los animales se pesaron semanalmente hasta el final del experimento (semana 12), calculándose la ganancia de peso individual semanal y total. El índice de conversión alimenticia resultó de dividir el consumo total de materia seca entre la ganancia de peso total en cada unidad experimental. El consumo se calculó diariamente a partir de la diferencia entre la materia seca ofrecida y rechazada en cada unidad experimental, donde el consumo para cada tratamiento correspondió a la suma de los consumos parciales del forraje y del bloque. Se determinó el consumo individual semanal y total.

Se calculó el mérito económico en base al costo del forraje y del bloque mineral, los consumos y la ganancia de peso en el experimento. El análisis estadístico de las respuestas en ganancia de peso, consumo de materia seca e índices de conversión se realizó a través de un análisis de varianza correspondiente a un diseño completamente al azar con dos tratamientos y cuatro repeticiones. Los datos fueron procesados mediante el procedimiento ANOVA del programa estadístico SAS $^{\circledR}$ (SAS Institute Inc., 1985). Todas las pruebas de hipótesis y de comparaciones de medias usaron un nivel de significancia de 0.05 .

\section{Resultados y Discusión}

La suplementación con bloques minerales incrementó la ganancia total de peso y redujo el índice de conversión alimenticia $(p<0.05$, Cuadro 4$)$, sin tener un efecto significativo sobre el consumo total de materia seca. Las diferencias en ganancia de peso entre tratamientos comenzaron a ser notorias a partir de la cuarta semana a favor del grupo de cuyes con suplementación mineral, de manera que se llegó a obtener 118 g más de ganancia de peso en el grupo suplementado; es decir, una diferencia de $25 \%$ de ganancia de peso. No obstante, los pesos finales fueron relativamente bajos desde el punto de vista comercial, posiblemente debido al bajo contenido de proteína del forraje (Cuadro 3). Los animales del tratamiento control presentaron signos de alimentación deficitaria, como pelo hirsuto, decaimiento y alopecia. 
Las diferencias en la ganancia de peso reflejan el efecto positivo de los minerales presentes en el bloque, los cuales en conjunto actúan como promotores de crecimiento (calcio, cobre, zinc y selenio) e intervienen directamente en el metabolismo energético (fósforo, yodo y magnesio) (Hintz, 1969). No obstante, Afuso (1976), suplementando calcio y fósforo, en una relación 2:1 mediante roca fosfatada y harina de huesos, no obtuvo respuestas en ganancia de peso e índice de conversión alimenticia ni en contenido de cenizas y P en el hueso. Tampoco hubo efecto en el estudio de González (1985), quien suplementó calcio en forma de cloruro sobre una dieta base de rye grass inglés a cuyes de dos meses. Es posible que la base forrajera usada en esos estudios haya suministrado el calcio y fósforo requerido por los animales. En el presente estudio, estos requerimientos (National Research Council, 1995) estaban cubiertos con el forraje suministrado, por lo que es posible que la respuesta positiva haya sido debida a la presencia en el bloque de otros minerales promotores del crecimiento.

No se encontró diferencias estadísticas significativas en el consumo total de materia seca, de modo que las diferencias en el índice de conversión alimenticia fueron debidas a las diferencias en ganancia de peso. El índice de conversión alimenticia se redujo de 6.9 a 5.5; es decir, una reducción de $26 \%$. Hay pocos trabajos en suplementación de cuyes donde se evaluó los cambios en conversión alimenticia como efecto de la suplementación de minerales; destacando los trabajos de Gamarra et al. (1989) e Hidalgo (1989), quienes suplementaron sulfato de cobre y sal de piedra sin encontrar diferencias entre tratamientos.

El costo del kilogramo de maíz chala fresco fue de $\mathrm{S} / .0 .30 / \mathrm{kg}$ y del bloque mineral fue de S/. 4.00/kg. El consumo de forraje fresco del T0 fue de $15.04 \mathrm{~kg}$ con un costo de S/. 4.51; en tanto que el consumo de forraje fresco y de bloque mineral en el T1 fue 14.2 y $0.316 \mathrm{~kg}$, respectivamente, con un cos- to total de S/. 5.52. En base a las ganancias de peso vivo por tratamiento (Cuadro 4), se encontró que por cada $100 \mathrm{~g}$ de ganancia de peso vivo se han invertido S/.1.26 en alimentos con el T0 y S/. 1.16 con el T1, indicando que salió $9 \%$ más económico suplementar a los cuyes con el bloque mineral.

Los resultados permiten concluir que existe un potencial de mejora productiva en la crianza de cuyes, particularmente en ganancia de peso y conversión alimenticia, por efecto de la suplementación mineral mediante bloques minerales.

\section{Literatura Citada}

1. Afuso A. 1976. Evaluación de la roca fosfatada de Bayóvar como fuente de fósforo en cuyes. Tesis de Ing. Zootecnista. Lima: Univ. Nacional Agraria La Molina. 83 p.

2. Becerra J, David, H. 1990. Observaciones sobre la elaboración y consumo de bloques de urea/melaza. Livestock Research for Rural Development 2(2). [Internet], [28 noviembre 2008]. Disponible en: http://www.lrrd.org/lrrd2/2/ becerra.htm

3. Chauca D. 1994. Fisiología digestiva de los cuyes. INIA Serie Didáctica 94. Lima, Perú. 11 p.

4. Chauca L. 1997. Producción de cuyes (Cavia porcellus). Organización de las Naciones Unidas para la Agricultura y la Alimentación. Estudio Técnico 138. Roma: FAO. p 1-32.

5. Díaz J. 1990. Utilización de cubos multinutricionales como suplemento en la alimentación de cuyes de engorde. Tesis de Ing. Zootecnista. Colombia: Universidad de Nariño. 23 p.

6. Gamarra JN, Florián A, Zaldívar MN. 1989. Suplementación mineral en la dieta de cuyes (Cavia porcellus) en crecimiento. En: Libro de Resúmenes XII Reunión Científica Anual de la Aso- 
ciación Peruana de Producción Animal. Lima, Perú.

7. González FR. 1985. Uso del cloruro de calcio en el engorde de cuyes. Tesis de Ing. Zootecnista. Huancayo: Univ Nacional del Centro del Perú. 57 p.

8. Hidalgo $\mathbf{M H}$. 1989. Efecto de la adición de sal negra y sulfato de cobre en el engorde de cuyes. Tesis de Ing. Zootecnista. Cerro de Pasco: Univ Nacional Daniel Alcides Carrión. 73 p.

9. Hintz B. 1969. Reingestion of feces in rodents and its daily rhythmicity. Cunicology 44: 82-97.

10. Hirikawa H. 2001. Coprophagy in leporids and other mammalian herbivores. Mammal Review 31: 61-80.

11. National Research Council. 1995. Nutrient requirements of laboratory animals $4^{\text {th }}$ ed. Washington DC: National Academic Press. 173 p.

12. Rivadeneira E. 1999. Engorde de cuyes usando alfalfa, bloques nutricio- nales, afrecho de trigo y concentrado comercial. Tesis de Médico Veterinario. Lima: Univ Nacional Mayor de San Marcos. 40 p.

13. Rueda E, de Combellas J. 1999. Evaluación de la suplementación con bloques multinutricionales en un sistema de producción ovina. I. Ovejas en lactancia. Rev. Fac. Agron. (LUZ) 16: 79-88.

14. Saravia J. 1994. Avances de investigación en la alimentación de cuyes. Estación Experimental La Molina. INIA. Lima. $62 \mathrm{p}$.

15. SAS Institute Inc. 1985. SAS/STAT Guide for personal computers. v 6. Cary, NC: SAS. 378 p.

16. Vargas, JE, Rivera JG. 1994. Efecto del bloque multinutricional sobre el comportamiento productivo y reproductivo en ovejas africanas. Livestock Research for Rural Development 6(2). [Internet], [25 noviembre 2008]. Disponible en: http:/ /www.lrrd.org/lrrd6/2/vargas2.htm 